\title{
Desenvolvimento da Habilidade de Comunicação dos Estudantes de Medicina para o Aconselhamento Pré-Teste HIV no Pré-Natal
}

\section{Development of Medical Students' Communication Skills for HIV Pre-Test Counseling at Prenatal Care}

\author{
Larissa Cynthia Cesar Rodrigues ${ }^{I}$ (iD \\ Reginaldo Antônio de Oliveira Freitas Júnior ${ }^{I, I I}$ (iD \\ Carolina Teixeira de Resende Barreto ${ }^{I}(\mathbb{D}$ \\ Alexandra do Nascimento Cassiano ${ }^{\text {II }}$ (D) \\ Alexandra Silva de Lima ${ }^{I}(\mathbb{D}$ \\ Carla Glenda Souza da Silva ${ }^{I}(\mathbb{D}$
}

\section{PALAVRAS-CHAVE}

- Profissionalismo.

- Competência Clínica.

- Cuidado Pré-Natal.

- Soropositividade para HIV.
Instituto Santos Dumont, Macaíba, Rio Grande do Norte, Brasil.

${ }^{\mathrm{II}}$ Universidade Federal do Rio Grande do Norte, Natal, Rio Grande do Norte, Brasil.

Introdução: As Diretrizes Curriculares Nacionais dos cursos de graduação em Medicina preconizam a ênfase na habilidade de comunicação para o profissionalismo do egresso. Promover o desenvolvimento da habilidade de comunicação efetiva para a competência clínica representa um desafio para a educação médica. O presente estudo objetivou contribuir para o desenvolvimento da habilidade de comunicação durante a formação médica por meio de estratégia de ensino e aprendizagem que utilizou como ferramenta o aconselhamento pré-teste HIV no pré-natal. Métodos: Trata-se de estudo com métodos mistos sequenciais e abordagem qualitativa, desenvolvido em três fases. Na primeira fase, 30 estudantes do internato em Tocoginecologia participaram de consulta simulada, gravada em áudio e vídeo, direcionada para conhecimentos específicos e habilidades para comunicação efetiva no tema. Na segunda fase, uma estratégia de feedback individual contou com a análise conjunta do vídeo e avaliação de critérios estabelecidos em checklist específico. Na terceira fase, buscouse avaliar a autopercepção dos estudantes acerca de sua participação na estratégia e de sua necessidade de desenvolvimento da habilidade de comunicação durante a formação. Resultados: A análise geral dos dados revela um baixo desempenho, sobretudo quanto aos conhecimentos específicos necessários à comunicação efetiva para o aconselhamento pré-teste HIV no pré-natal. Conclusões: Nesse sentido, a autopercepção dos estudantes mostrou-se coerente com os resultados observados e apontou a necessidade de melhorar os conhecimentos específicos aliados às habilidades de comunicação para, então, possibilitar um aconselhamento adequado. Além disso, nos discursos dos estudantes, são frequentes falas reflexivas a respeito da importância de praticar e desenvolver a habilidade de comunicação. 


\section{KEY-WORDS}

- Professionalism.

- Clinical Competence.

- Prenatal Care.

- HIV Seropositivity.

\section{ABSTRACT}

Introduction: The Brazilian National Curriculum Guidelines for medical undergraduate courses underscore the emphasis on communication skills for recently graduated professionals. Promoting the development of effective communication skills for clinical competence poses a challenge for medical education. The present study aims to contribute to the development of communication skills during medical education through a teaching and learning strategy that uses prenatal HIV pretest counseling as a tool. Methods: This is a study with sequential mixed methods and qualitative approach developed in three phases. In the first phase, thirty students from the Tocho-Gynecology Internship participated in a simulated consultation, which was recorded in audio and video, directed to specific knowledge and skills for effective communication in the topic. In the second phase, an individual feedback strategy included the joint analysis of the video and the evaluation of established criteria in a specific checklist. In the third phase, the objective was to evaluate the students' self-perception about their participation in the strategy and their need to develop communication skills during medical training. Results: The general analysis of the data reveals a poor performance, especially regarding the specific knowledge required for effective communication for prenatal HIV pretest counseling. Conclusion: Therefore, the students' self-perception was consistent with the observed results and indicated the need to improve specific knowledge combined with communication skills, so as to allow appropriate counseling. Moreover, the students' discourses frequently disclose reflective comments about the importance of practicing and developing communication skills.

Recebido em 22/10/19

Aceito em 25/11/19

\section{INTRODUÇÃO}

A efetividade do cuidado em saúde pressupõe a efetividade da interação que deve ser estabelecida entre quem cuida e quem é cuidado. Os resultados esperados desse cuidado em saúde serão influenciados sobremaneira pelo modo como o paciente e o médico mantêm um processo efetivo de comunicação entre si. Dessa forma, reconhece-se que o médico precisa de um conjunto mínimo de habilidades para estabelecer uma comunicação efetiva que conduza uma consulta na direção do cuidado mais adequado ${ }^{1}$.

Em meio aos extraordinários avanços técnicos e científicos, tem sido comprovada a relação positiva entre a comunicação efetiva na atenção à saúde e a recuperação mais rápida de pessoas com determinadas doenças, resultando em melhor qualidade de vida e maior satisfação dos envolvidos. A habilidade de comunicação também se mostra essencial nos processos de promoção da saúde e prevenção de doenças².

Pode-se afirmar que grande parte da chamada crise no setor da saúde nasce de limitações dos profissionais quanto à habilidade de comunicação com aqueles que são o alvo primordial de suas ações ${ }^{3}$. De fato, um estudo de 2007 realizado no Canadá mostrou que as notas baixas relativas ao quesito comunicação em exames para licenciamento médico anunciaram, nos anos seguintes, o aumento das reclamações de pacientes contra médicos às autoridades de regulação médica ${ }^{4}$.

O profissionalismo também é importante e envolve competências que capacitam os médicos a servir aos interesses dos pacientes acima dos seus próprios, exercendo altruísmo, responsabilidade social, excelência, dever, serviço, honra, integridade e respeito pelos outros. Assim, o profissionalismo estabelece e mantém padrões de competência e integridade ${ }^{5}$.

Em 2014, as Diretrizes Curriculares Nacionais (DCN) dos cursos de graduação em Medicina reforçaram a importância das competências de comunicação e profissionalismo. As DCN estabelecem que a educação médica deve buscar garantir para o futuro exercício profissional do médico que este seja hábil em concretizar a comunicação, por meio de linguagem verbal e não verbal, com usuários, familiares, comunidades e membros das equipes profissionais, com empatia, sensibilidade e interesse, preservando a confidencialidade, a compreensão, a autonomia e a segurança da pessoa sob cuidado ${ }^{6}$.

Há evidências sustentando que a habilidade de comunicação influencia grandemente o raciocínio clínico. E o contrário também é verdadeiro: o raciocínio clínico tem uma influência considerável na comunicação clínica. Integrar a comunicação clínica com o processo de construção do raciocínio clínico talvez seja uma boa forma de abordar a habilidade de comunicação no currículo médico ${ }^{7}$.

O aconselhamento pré-teste HIV refere-se ao diálogo existente, antes da realização do exame, entre o profissional de saúde e a pessoa a quem foi ofertado o teste de HIV. Caracteriza-se como um processo baseado na escuta ativa, por meio do estabelecimento de uma relação de confiança entre profissional e usuário, de modo a fornecer a este os elementos necessários para que ele possa se reconhecer como agente de sua condição de saúde. Constitui-se na troca de informações, suporte emocional, orientação e avaliação de riscos, possibilitando que o usuário busque soluções realísticas para enfrentar seus problemas relacionados às infecções sexualmente transmissíveis (IST), à infecção pelo vírus da imunodeficiência humana (human immunodeficiency virus - HIV) e à síndrome da imunodeficiência adquirida (acquired immodeficiency syndrome-Aids) ${ }^{8}$.

Em 2005, o Ministério da Saúde lançou a Portaria $\mathrm{n}^{\circ} 34^{9}$ que regulamenta o uso de testes rápidos para diagnósticos de HIV. A implementação do teste rápido se justifica por ser uma estratégia eficaz e confiável, oferecida de forma ampla à população, por meio do Sistema Único de Saúde (SUS), ofertado de forma imediata e com tempo reduzido de espera para o resultado. $\mathrm{O}$ teste rápido não necessita de estrutura laboratorial complexa e é feito a partir da coleta de uma gota de sangue, e seu resultado é obtido em até 30 minutos. Sua realização inclui o aconselhamento como uma de suas etapas ${ }^{10}$. Estudos nacionais

REVISTA BRASILEIRA DE EDUCAÇÃO MÉDICA

2 44 (1) : e026; 2020 
sobre aconselhamento para gestantes e parturientes criticam como este tem sido realizado, na medida em que, majoritariamente, restringe-se a informar sobre o diagnóstico de IST no pré e pós-parto, com pouca atenção à autonomia das mulheres no processo decisório de testagem, por exemplo ${ }^{11,12}$. Pesquisadores ressaltam a importância de aperfeiçoar a escuta dos profissionais, enfatizando que o aconselhamento pré-teste não se reduz à obtenção do consentimento para a efetuação do exame ${ }^{13}$.

Por proporcionar ao paciente acesso imediato ao diagnóstico, o aconselhamento pré-teste HIV exemplifica uma situação na qual a comunicação efetiva assume caráter especialmente determinante da qualidade do cuidado e da segurança da paciente, sobretudo pelo potencial dano súbito que um resultado positivo pode acarretar e por se tratar de exame já incorporado na propedêutica laboratorial do pré-natal.

O profissional que realiza o pré-natal deve permitir que a usuária expresse suas preocupações e angústias, garantindo a atenção resolutiva e possibilitando o vínculo da gestante com a equipe de saúde. O diálogo franco, a sensibilidade e a capacidade de percepção desse profissional são condições básicas para que o saber em saúde seja colocado à disposição da mulher e da sua família - atores principais da gestação e do parto ${ }^{8}$.

O desenvolvimento da habilidade de comunicação efetiva para a competência clínica representa um desafio para a educação médica. Desse modo, o presente estudo objetivou contribuir para o desenvolvimento dessa habilidade durante a formação médica por meio de estratégia de ensino e aprendizagem que utilizou como ferramenta disparadora o aconselhamento para a realização do teste anti-HIV no pré-natal.

\section{METODOLOGIA}

O percurso metodológico adotado foi uma pesquisa com abordagem qualitativa, sendo desenvolvido um estudo com métodos mistos sequenciais. A população do estudo foi composta por estudantes do sexto ano do curso de Medicina da Universidade Federal do Rio Grande do Norte (UFRN) que cumpriam regularmente o internato de Tocoginecologia II e concordaram em participar do estudo.

A coleta de dados ocorreu no período de abril a agosto de 2018, após o projeto ser aprovado pelo Comitê de Ética em Pesquisa do Hospital Universitário Onofre Lopes/UFRN, conforme Parecer no 2.578.693, e os participantes preencherem os termos de autorização para gravação de imagem e voz e de consentimento livre e esclarecido.

Desse modo, incluímos três fases no estudo, desenvolvidas de forma integrada: avaliação inicial do estudante sobre os conhecimentos e as habilidades para o aconselhamento pré-teste HIV no pré-natal, feedback individualizado usando checklist específico e avaliação da autopercepção dos estudantes sobre sua participação na estratégia de ensino-aprendizagem.

Fase I: Avaliação inicial do estudante sobre os conhecimentos e as habilidades para o aconselhamento pré-teste HIV no pré-natal

A coleta de dados iniciou-se com a participação do aluno em uma "consulta simulada" gravada em áudio e vídeo, reproduzindo situação de aconselhamento pré-teste HIV durante a primeira consulta do pré-natal, com paciente padronizada e simulada, no início do rodízio do estágio supervisionado no Centro de Educação e Pesquisa em Saúde (Ceps) Anita Garibaldi. Os alunos foram orientados a realizar um aconselhamento préteste de HIV a uma gestante que chegava à sua primeira consulta de prénatal, tendo prévio conhecimento de que, de fato, tratava-se de uma atriz.
O tempo disponível para execução dessa tarefa foi de até 20 minutos. A atividade não fez parte da avaliação final da disciplina e nem foi obrigatória.

As atrizes que realizaram o papel de gestantes eram voluntárias e foram treinadas para seguir um roteiro de falas previamente elaborado. O script considera esclarecimentos rotineiros que devem constar no aconselhamento pré-teste de HIV, segundo as diretrizes do Programa Nacional de IST/Aids ${ }^{8}$.

As simulações aconteceram nas salas de ambulatório do pré-natal que são comumente usadas durante o internato, sendo, portanto, ambiente com o qual os estudantes já estavam familiarizados. Nelas, estavam presentes o aluno, a paciente-atriz, o responsável por filmar a atividade previamente apresentado ao estudante e o avaliador, portando o checklist de avaliação, em posição não visível para o aluno.

O referencial teórico que subsidiou a construção do checklist foi as diretrizes preconizadas pelo Ministério da Saúde acerca daquilo que deve ser efetivamente abordado no aconselhamento pré-teste HIV ${ }^{14}$.

O checklist conteve 26 itens de avaliação, sendo 13 referentes à dimensão "conhecimentos" e 13 referentes à dimensão "habilidades" necessários à comunicação efetiva para o aconselhamento pré-teste HIV no pré-natal. Os 26 itens foram agrupados em cinco seções: acolhimento inicial, identificação da razão do teste, conversa sobre a doença, possíveis resultados e rapport. Cada item foi avaliado quanto à execução dele pelo aluno, segundo os critérios "sim" e "não". Os estudantes não conheciam o checklist antes da atividade.

\section{Fase II: Feedback individualizado usando checklist específico}

O feedback individual foi baseado na avaliação conjunta (avaliador e aluno) do vídeo da consulta simulada, tendo o checklist como ferramenta objetiva e obedecendo aos pressupostos estabelecidos pelas Diretrizes Nacionais para o Aconselhamento em Infecção Sexualmente Transmissível, Infecção pelo Vírus da Imunodeficiência Humana e Hepatites Virais (IST/HIV/HV), preconizados pelo Ministério da Saúde ${ }^{8}$.

O vídeo foi assistido pelo estudante e pelo avaliador, simultaneamente, acompanhando o checklist e observando os respectivos acertos e erros. Incluir o estudante na análise do vídeo e na avaliação dos critérios estabelecidos no checklist, numa espécie de autoavaliação, objetivou o caráter formativo da estratégia, estimulando a habilidade reflexiva sobre as necessidades de aprendizagem e ajudando o estudante a identificar as mudanças necessárias antes do fim daquele rodízio de aprendizado.

Para fins de análise dos resultados das fases I e II, as avaliações de desempenho foram calculadas a partir dos registros dos checklists, tendo como base os itens avaliados em cada dimensão. Para cada item, utilizaram-se escores binários, em que o comportamento realizado pelo aluno obteve pontuação de 1 ou 0 , caso tivesse obtido ou não o desempenho esperado, respectivamente. $\mathrm{O}$ total de pontos e percentagens foi calculado para determinar o desempenho.

Os dados gerados pela pesquisa foram codificados e registrados em planilha Microsoft Office Excel 2003. Em seguida, construíram-se bancos de dados associados a programas de análise estatística como o software Statistical Package for the Social Sciences.

Fase III: Avaliação da autopercepção dos estudantes sobre sua participação na estratégia de ensino-aprendizagem

Essa fase do estudo buscou avaliar a perspectiva dos estudantes

REVISTA BRASILEIRA DE EDUCAÇ̃̃o MÉDICA

3 44 (1) : e026; 2020 
acerca de como suas percepções e seus pressupostos sobre a estratégia se integrariam às necessidades de desenvolvimento da habilidade de comunicação durante a formação médica. Após todos os participantes concluírem o internato em Tocoginecologia II, foram convidados, por ligação telefônica, a contribuir para o estudo respondendo a um questionário semiestruturado enviado por e-mail, pela ferramenta survey on-line, com a utilização do software Survey Monkey.

O questionário, contendo cinco perguntas, buscou avaliar, na primeira, a percepção dos estudantes sobre os aspectos relacionados ao grau de satisfação em ter participado da atividade, grau de satisfação com o próprio desempenho no aconselhamento e grau de estresse sentido durante a atividade, por meio de análise descritiva das frequências de respostas de acordo com a escala de Likert com 5 graus, sendo 1 o grau mínimo, e 5, o grau máximo.

$\mathrm{Na}$ segunda questão, a escala de Likert com cinco conceitos (1 péssimo, 2 - ruim, 3 - regular, 4 - bom e 5 - ótimo) foi utilizada para avaliar o grau de satisfação dos alunos com relação à qualidade do feedback e aos comentários finais recebidos sobre seu desempenho na realização do aconselhamento pré-teste HIV na primeira consulta pré-natal ${ }^{15}$.

$\mathrm{Na}$ terceira questão, os estudantes foram solicitados a refletir sobre três aspectos que consideraram ter aprendido ao participarem da atividade e hierarquizá-los em ordem de importância.

Por fim, uma vez que se trata da "satisfação" sobre uma dimensão altamente subjetiva, o questionário semiestruturado também objetivou a abordagem qualitativa por meio de duas questões abertas, em que se utilizaram os seguintes estímulos: aspectos positivos sobre a atividade (“O que de fato foi bom e relevante para a sua formação?") e aspectos negativos sobre a atividade (“O que poderia ter sido melhor?").

Para análise das respostas obtidas no questionário semiestruturado, adotou-se a técnica da análise de conteúdo temática categorial referenciada por $\operatorname{Bardin}^{16}$.

Para auxiliar a apresentação dos dados, empregou-se a técnica nuvem de palavras, criada a partir do software WordleTM. Nuvem de palavras é uma ferramenta virtual que mostra a frequência com que as palavras aparecem em um dado contexto. Nessa ferramenta, utilizam-se tamanhos de fontes de letras diferentes de acordo com as ocorrências das palavras no texto analisado.

\section{RESULTADOS E DISCUSSÃO}

Nas fases I e II, participaram 30 estudantes, e os respectivos checklists oriundos de seus desempenhos na prática das consultas simuladas constituíram o objeto de análise.

A Tabela 1 apresenta a média, a mediana, o mínimo, o máximo e o percentual de desempenho (acertos) dos alunos nas dimensões "conhecimentos" e "habilidades" e no total dos itens avaliados.

No contingente de 30 estudantes que participaram da consulta simulada e do total dos 26 itens do checklist, a mediana de acertos foi de 11,0 $\pm 2,86$ itens, com mínimo de sete e máximo de 18 itens com avaliação "sim", o que significa um percentual médio de desempenho de $45 \%$ de acertos. Para a dimensão "conhecimentos" (13 itens), a mediana de acertos foi de 4,0 \pm 1,60 itens, com mínimo de dois e máximo de nove itens e $36,1 \%$ de percentual médio de desempenho satisfatório. Já para a dimensão "habilidades" (13 itens), a mediana de acertos foi de 7,0 \pm 1,76 itens com mínimo de três e máximo de dez acertos e percentual médio de desempenho satisfatório de $53,8 \%$.
A análise geral dos dados obtidos com a realização da consulta simulada revela um baixo desempenho dos estudantes avaliados, notadamente no que concerne aos conhecimentos específicos necessários à comunicação efetiva para o aconselhamento pré-teste HIV no pré-natal. Em um estudo brasileiro que avaliou as atitudes e o conhecimento de $85 \%$ dos obstetras das maternidades públicas da cidade de Salvador ( $\mathrm{n}$ = 129) sobre as recomendações do Ministério da Saúde para a profilaxia da transmissão vertical do HIV, enquanto $97,6 \%$ desses profissionais concordaram com a oferta de testagem rápida, apenas $58,9 \%$ o fizeram em relação ao aconselhamento pré-teste ${ }^{17}$. Trata-se de uma importante questão que emerge no presente estudo e que parece também existir na realidade dos profissionais que já atuam no sistema de saúde.

Embora globalmente baixo, também se observa desempenho relativamente melhor nos itens relativos à dimensão "habilidades", notadamente relacionado aos acertos nos itens que compunham a seção rapport. Ou seja, proporcionalmente, os alunos acertaram mais os quesitos referentes às habilidades reconhecidas como necessárias para a comunicação efetiva do que aqueles relacionados aos conhecimentos específicos sobre o tema para a qualidade do aconselhamento.

$\mathrm{Na}$ fase III, buscou-se analisar a percepção dos estudantes quanto à integração da estratégia às necessidades de desenvolvimento da habilidade de comunicação durante a formação médica. Dos 30 alunos que participaram da fase I, 13 (43,3\%) responderam ao questionário semiestruturado.

No Gráfico 1, são apresentadas as respostas relativas ao grau de satisfação do estudante quanto a ter participado da atividade, ao grau de satisfação com seu desempenho no aconselhamento e ao grau de estresse sentido durante a atividade. Observa-se que a maioria dos estudantes (53,85\%) se mostrou satisfeita e sentiu nível de estresse mediano (grau 3) em relação à sua participação na atividade. Destaca-se o elevado grau de satisfação com o próprio desempenho, embora seja considerado baixo o desempenho global dos avaliandos quanto à comunicação para o aconselhamento pré-teste.

A escala de Likert com cinco conceitos foi utilizada para avaliar o grau de satisfação dos alunos com relação à qualidade do feedback. No Gráfico 2, são apresentadas as repostas e a totalidade dos estudantes que classificou a abordagem empregada como sendo boa ou ótima. Os resultados observados sugerem a potencialidade da estratégia adotada no presente estudo para o processo de avaliação formativa, sobretudo para o estímulo à habilidade reflexiva.

$\mathrm{Na}$ avaliação qualitativa sobre os aspectos considerados aprendidos durante atividade, destacamos as palavras expressas na Figura 1.

\begin{tabular}{|c|c|c|c|c|c|}
\hline \multicolumn{6}{|c|}{$\begin{array}{c}\text { Tabela } 1 \\
\text { Média, mediana, mínimo, máximo e percentual de desempenho } \\
\text { satisfatório dos estudantes que participaram da consulta simulada, } \\
\text { de acordo com a avaliação do checklist. }\end{array}$} \\
\hline $\begin{array}{l}\text { Dimensões } \\
\text { avaliadas }\end{array}$ & Média & Mediana & Mínimo & Máximo & $\begin{array}{c}\text { Desempenho } \\
\%\end{array}$ \\
\hline $\begin{array}{c}\text { Dimensão } \\
\text { Conhecimentos }\end{array}$ & 4,7 & 4,0 & 2,0 & 9,0 & $36,1 \%$ \\
\hline $\begin{array}{c}\text { Dimensão } \\
\text { Habilidades }\end{array}$ & 7,0 & 7,0 & 3,0 & 10,0 & $53,8 \%$ \\
\hline Total & 7,0 & 11,0 & 7,0 & 18,0 & $45 \%$ \\
\hline
\end{tabular}

Elaborada pelos autores

REVISTA BRASILEIRA DE EDUCAÇÃo MÉDICA

\begin{tabular}{l}
44 (1) : e026; 2020 \\
\hline
\end{tabular} 
A palavra "conhecimento" assumiu expressiva importância no discurso dos participantes do estudo. Nesse sentido, a autopercepção dos estudantes mostrou-se coerente com os resultados objetivos observados na avaliação da fase I. Reconhecer a aquisição de conhecimentos como aspecto de destaque no aprendizado mediado pela estratégia empregada pode estar relacionado à identificação de lacunas importantes quanto aos conhecimentos específicos necessários para o aconselhamento pré-teste HIV no pré-natal. Por sua

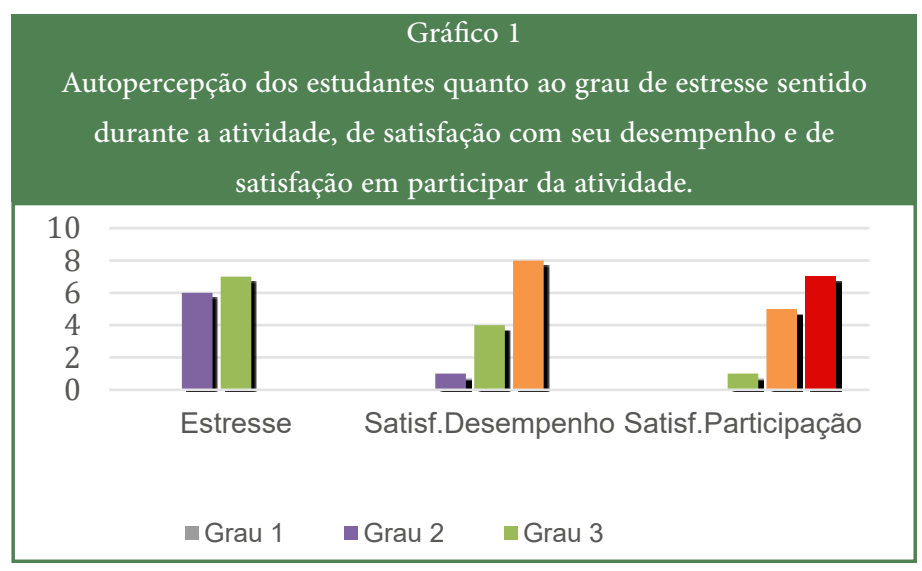

Elaborado pelos autores

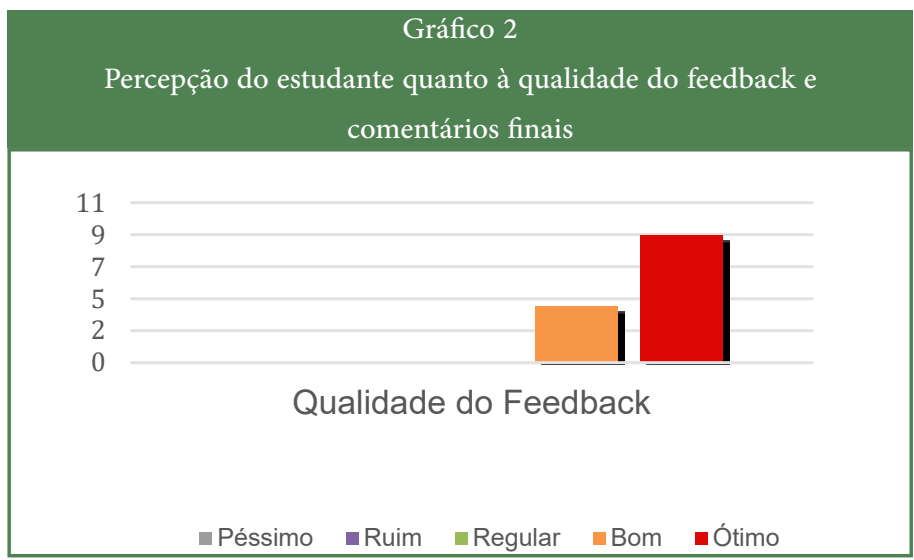

Elaborado pelos autores

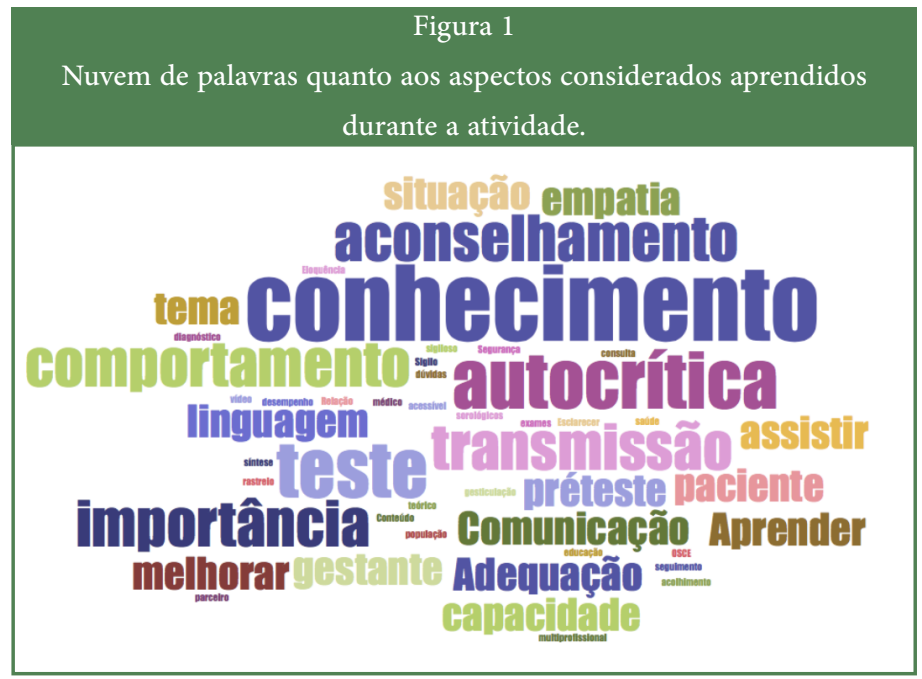

Elaborada pelos autores vez, a frequência com que expressões como "autocrítica", "comportamento", "aconselhamento", "capacidade" e "empatia" aparecem nos discursos chama a atenção para o potencial da estratégia empregada como disparadora da habilidade reflexiva do aluno sobre a sua práxis.

A partir da leitura flutuante desse conjunto de falas, segundo a técnica da análise de conteúdo temática categorial, para análise qualitativa, sustentam-se três categorias temáticas: habilidade de comunicação, conhecimento e habilidade reflexiva.

Em um total de 38 citações, observa-se que 18 enfatizaram as habilidades de comunicação como aspecto mais aprendido na atividade, 15 destacaram o conhecimento e cinco mencionaram itens referentes à habilidade reflexiva como ponto interessante ao aprendizado.

Foi interessante constatar a frequência com que as falas destacavam a comunicação ou algum aspecto ligado a ela. Isso evidencia que uma atitude reflexiva foi despertada nos estudantes acerca dessa habilidade, reforçando que estratégias assim merecem replicação. Entre as unidades de análise dos discursos individuais, destacam-se algumas que são consideradas capazes de traduzir esse pensamento (Quadro 1).

Sobre os aspectos positivos detectados em relação à atividade, utilizando como estímulo "O que de fato foi bom e relevante para a sua formação?", destacam-se as palavras expressas na Figura 2.

\begin{tabular}{|c|c|}
\hline \multicolumn{2}{|c|}{$\begin{array}{l}\text { Exemplos de Unidades de Análise, por Categorias Temáticas, } \\
\text { quanto aos aspectos aprendidos na atividade pelos alunos em } \\
\text { relação à consulta simulada }\end{array}$} \\
\hline Categorias & Unidades de Análise \\
\hline $\begin{array}{l}\text { Habilidades de } \\
\text { Comunicação }\end{array}$ & $\begin{array}{l}\text { Adequação da linguagem técnica à população leiga. } \\
\text { Demonstrar empatia. } \\
\text { Formas de abordagem do tema aconselhamento com } \\
\text { o paciente. } \\
\text { Capacidade de síntese. } \\
\text { Percebi que tenho boa gesticulação e empatia durante } \\
\text { a consulta. } \\
\text { Aprendi como me comportar frente aos } \\
\text { questionamentos do paciente. } \\
\text { Assistir a mim mesma e ver como eu me comportei } \\
\text { diante da situação e como poderia melhorar. } \\
\text { Tentar ser mais clara nas informações transmitidas } \\
\text { ao paciente. }\end{array}$ \\
\hline Conhecimento & $\begin{array}{l}\text { Sequência de informações que necessitam ser } \\
\text { passadas ao paciente. } \\
\text { A importância da educação em saúde para a } \\
\text { população. } \\
\text { Tópicos essenciais a serem abordados no } \\
\text { aconselhamento pré-teste. } \\
\text { Orientação sobre importância dos exames } \\
\text { sorológicos. } \\
\text { Conteúdo teórico. }\end{array}$ \\
\hline Habilidade Reflexiva & $\begin{array}{l}\text { Fazer uma autocrítica a respeito do meu desempenho } \\
\qquad \text { a partir do vídeo. } \\
\text { Autopercepção dos conhecimentos sobre o tema. } \\
\text { Autoavaliação sobre a capacidade de transmitir } \\
\text { informações de modo acessível. }\end{array}$ \\
\hline
\end{tabular}

Elaborado pelos autores 


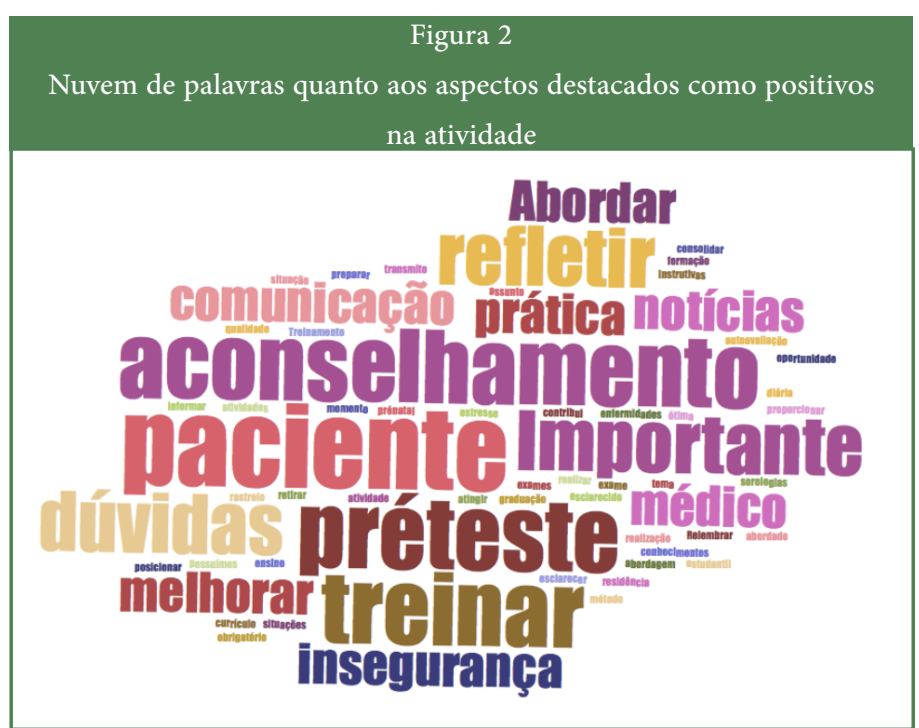

Elaborada pelos autores

Observa-se o destaque dado às palavras "aconselhamento", "paciente", "importante" e "treinar". Como aspectos positivos, em suas falas, os estudantes destacaram a importância da atividade por fazê-los vivenciar um momento de grande necessidade de comunicação efetiva que ainda é pouco abordado na academia. Além da atividade funcionar como um "treino para atender a paciente", também se mostrou relevante quando evocou conhecimentos importantes cuja aplicabilidade prática para o aconselhamento e para a qualidade do cuidado não havia sido reconhecida pela maioria dos alunos participantes do estudo.

Para a análise qualitativa desses aspectos considerados positivos, sustentam-se as mesmas três categorias temáticas utilizadas anteriormente: habilidade de comunicação, conhecimento e habilidade reflexiva. Em um total de 12 citações, observa-se que seis enfatizaram as habilidades de comunicação como aspecto positivo da atividade, cinco destacaram o conhecimento e uma mencionou a habilidade reflexiva como ponto positivo para o aprendizado.

Observar que a habilidade de comunicação foi praticamente tão citada quanto o conhecimento evoca, mais uma vez, o reconhecimento do estudante quanto à necessidade de ter o saber teórico específico para aliálo às habilidades de comunicação e, então, possibilitar um aconselhamento pré-teste HIV adequado. Comprova-se isso nas falas que mais se destacam segundo o Quadro 2.

Considerando os aspectos negativos encontrados na atividade, usou-se o estímulo "O que poderia ter sido melhor?", e as palavras mais expressas estão na Figura 3.

Nessa nuvem, observam-se, principalmente, as palavras "dois tempos", "câmera", "aula prévia" e "planejamento" que foram a expressão dos pontos negativos da atividade citados pelos alunos. Eles destacaram o fato de estarem sendo observados e filmados como causador de inibição. Mostraram-se também insatisfeitos com a falta de aula ou roteiro escrito sobre aconselhamento pré-teste HIV antes da prática, já que não tinham conhecimento prévio adequado, o que foi considerado por eles como ausência de planejamento da atividade.

Para a análise qualitativa desses aspectos considerados negativos, agrupamos três categorias temáticas: ser observado, planejamento e conhecimento.

\begin{tabular}{|c|c|}
\hline $\begin{array}{l}\text { Exemplos de U } \\
\text { quanto aos aspect }\end{array}$ & $\begin{array}{l}\text { Quadro } 2 \\
\text { nidades de Análise, por Categorias Temáticas, } \\
\text { s considerados positivos pelos alunos em relação } \\
\text { à consulta simulada }\end{array}$ \\
\hline Categorias & Unidades de Análise \\
\hline $\begin{array}{l}\text { Habilidades de } \\
\text { Comunicação }\end{array}$ & $\begin{array}{l}\text { Serviu como referência inclusive para comunicação } \\
\text { de más notícias, já que temos poucas atividades } \\
\text { instrutivas como essa durante a graduação. } \\
\text { Treinar a realização do aconselhamento pré teste na } \\
\text { paciente, algo que não costumamos fazer no dia a dia } \\
\text { da nossa formação. } \\
\text { Foi importante para termos um momento para } \\
\text { treinar um aconselhamento pré teste, já que no dia a } \\
\text { dia isso pouco é feito. }\end{array}$ \\
\hline Conhecimento & $\begin{array}{l}\text { Importante para nós treinarmos para uma situação } \\
\text { que com certeza viveremos como médicos, além de } \\
\text { nos treinar para prova de residência e ser uma ótima } \\
\text { oportunidade de consolidar nossos conhecimentos. } \\
\text { Treinamento referente à forma de abordagem das } \\
\text { sorologias, o qual não possuímos no currículo } \\
\text { obrigatório, e que nos deparamos com grande } \\
\text { frequência na prática diária, tanto estudantil quanto } \\
\text { médica. } \\
\text { Ver que eu não lembrava de algumas informações } \\
\text { questionadas. }\end{array}$ \\
\hline Habilidade Reflexiva & $\begin{array}{l}\text { A auto avaliação proporcionada pelo método } \\
\text { contribui para melhorar a comunicação com o } \\
\text { paciente. }\end{array}$ \\
\hline
\end{tabular}

Elaborado pelos autores

\section{Figura 3}

Nuvem de palavras quanto aos aspectos destacados como negativos na atividade.

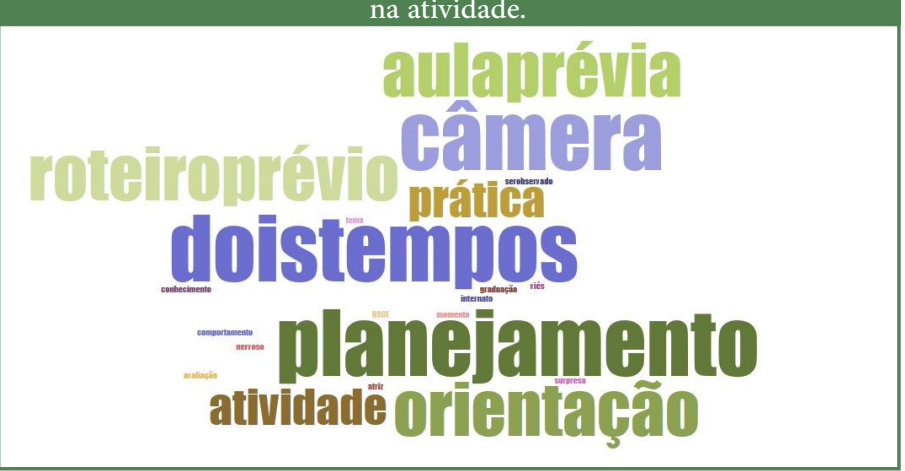

Elaborada pelos autores

Em um total de 12 citações, três enfatizaram ser observado como aspecto negativo da atividade, cinco mencionaram um déficit no planejamento dela e quatro destacaram alguma limitação do conhecimento como ponto negativo.

Convém esclarecer que os alunos já tiveram acesso aos conhecimentos sobre o aconselhamento pré-teste HIV pelo menos em quatro oportunidades no seu currículo obrigatório: quando acadêmicos das disciplinas de Ginecologia e Obstetrícia e Infectologia e quando cumpriram o internato em Tocoginecologia I e Saúde Coletiva. Acreditase que a formação acadêmica falhe em não promover uma adequada 
conexão entre esses conhecimentos e sua aplicabilidade na prática do aconselhamento. O Quadro 3 destaca as principais falas.

A habilidade de comunicar-se efetivamente representa aspecto central para o desenvolvimento de competências profissionais na formação médica. Temas bastante atuais nas discussões sobre educação para as profissões da saúde, como competência cultural, profissionalismo, metodologias ativas de ensino-aprendizagem, educação interprofissional e social accountability, têm na habilidade de comunicação uma grande área de intercessão de interesses.

Incentivar o investimento em estratégias que primem pelo desenvolvimento da habilidade de comunicação parece sensato e necessário quando se constata que as palavras mais mencionadas pelos estudantes, dentro dos aspectos que eles disseram aprender ao participarem da atividade, foram "conhecimento", "autocrítica", "aconselhamento" e "comportamento".

Para Freitas Júnior et al.18, a habilidade de comunicação efetiva é essencial para a compreensão conceitual sobre competência cultural para as profissões da saúde. Esta última congrega habilidades de comunicação efetiva, a despeito da diversidade linguística, acessando e compreendendo o sistema de crenças e práticas das pessoas às quais o cuidado em saúde se destina. Por sua vez, o desenvolvimento de competência cultural revelase indissociável do pressuposto da responsabilidade social no contexto da educação para as profissões da saúde. Nesse sentido, preocupar-se com a formação de profissionais capazes de interagir eficazmente com indivíduos e populações culturalmente diferentes transcende a dimensão curricular do perfil do egresso desejado pelas escolas de saúde para alcançar a dimensão da efetividade dos sistemas de saúde e da necessidade de que estes sejam inclusivos, democráticos e equânimes.

\section{Quadro 3}

Exemplos de Unidades de Análise, por Categorias Temáticas,

quanto aos aspectos considerados negativos pelos alunos em relação

\section{à consulta simulada}

\section{Categorias}

Ser Observado

Saber que está sendo observado causa viés no nosso comportamento.

Deveria esconder a câmara, pois deixa o avaliado mais nervoso.

Dispensar o operador de câmera (deixar só a câmera no pedestal)

Conhecimento Poderia haver uma espécie de aula ou orientação melhor sobre o assunto antes, porque nunca tivemos uma boa orientação sobre esse assunto na nossa prática e dessa forma a atividade seria mais instrutiva. Deveria estar no planejamento.

Deveria te algum roteiro ou aula prévia, deveria planejar isso.

Poderia haver uma orientação por escrito descrevendo o que se espera avaliar na atividade.

Planejamento Acredito que poderia ser feito em outro momento do internato ou graduação para que a próxima fase pudesse ser feita no período da nossa passagem pelo Anita. Poderia utilizar um usuário para a avaliação.

Elaborado pelos autores
O presente estudo compartilha a concepção da importância que a comunicação efetiva deve assumir durante a formação médica e buscou contribuir para o desenvolvimento dessa habilidade, valendose do aconselhamento pré-teste para o HIV durante o pré-natal como ferramenta de ensino e aprendizagem. É interessante verificar que 53,85\% dos alunos que responderam ao questionário mostraram-se satisfeitos em grau máximo em participar da atividade. Embora a mesma percentagem de estudantes tenha dito que vivenciou um nível de estresse mediano (nota 3), eles puderam perceber na atividade pontos positivos para a formação, expressos nas palavras mais mencionadas: "aconselhamento", "importante", "refletir" e "treinar". Isso pode revelar que o estudante tem o real desejo de incrementar sua própria formação ao valorizar estratégias que incentivam o aprimoramento da habilidade de comunicação.

O curso de Medicina da UFRN oferta algumas disciplinas que valorizam o aperfeiçoamento da habilidade de comunicação. A optativa "Medicina e Arte" tem carga horária de 30 horas e conta com três avaliações. A disciplina objetiva, por meio da discussão sobre as diversas manifestações artísticas em suas interfaces com a medicina e saúde, fortalecer a formação humanística e ética dos estudantes. Para Mangione et al. ${ }^{19}$, a exposição dos alunos do curso médico à literatura, à música, ao teatro e às artes visuais relaciona-se à aquisição de importantes qualidades médicas, principalmente tolerância a ambiguidades, empatia e sabedoria. Desse modo, as discussões nessa disciplina pretendem estimular a iniciativa artística como elemento essencial à manutenção da sensibilidade do profissional de saúde.

A atividade extracurricular "Comunicação médico-paciente: a arte de dar más notícias" é desenvolvida pelo Programa de Ensino Tutorial e conta com carga horária de 60 horas. Entre os objetivos, destacam-se: 1. desenvolver a habilidade de comunicação na relação médico-paciente por meio do uso do protocolo Spikes que descreve, de maneira didática, seis passos para comunicar más notícias e 2. proporcionar momentos de atividade prática em comunicação dentro das diversas especialidades clínicas com discussões e reflexões sobre situações-problema ${ }^{20}$.

A disciplina optativa "Comunicação e relação interpessoal na prática médica” tem carga horária de 30 horas e conta com três avaliações. Os principais objetivos da disciplina são: reconhecer as características individuais de cada paciente e a forma mais adequada de comunicar-se com ele; vivenciar e identificar os aspectos emocionais, éticos, implícitos na relação médico-paciente; e desenvolver a necessidade de buscar atitudes médicas empáticas, solidárias e objetivas.

"Competência cultural na atenção à saúde da mulher quilombola" também é uma disciplina optativa, tem carga horária de 60 horas e conta com três avaliações. A disciplina objetiva proporcionar aos estudantes a vivência integrada de conhecimentos, habilidades e atitudes necessários para o desenvolvimento de competências culturais no contexto da atenção à saúde e educação de populações cultural e etnicamente diversas. $\mathrm{O}$ elemento de habilidades se concentra nas habilidades de comunicação e compreensão dos determinantes sociais, culturais e comportamentais do processo saúde-doença das mulheres quilombolas.

Diante dessas oportunidades de exposição ao assunto comunicação e saúde, observa-se que o estudante já consegue privilegiar certos itens relativos à dimensão "habilidades", principalmente no que concerne ao comportamento e às atitudes diante do paciente (rapport). No entanto, além do aprimoramento dessas habilidades, é necessário conectá-las ao

7 REVISTA BRASILEIRA DE EDUCAÇÃO MÉDICA

7 44 (1) : e026; 2020 
conhecimento teórico adequado que capacita para a realização de um aconselhamento ideal.

O entendimento de que uma boa comunicação com pacientes e famílias pode ser ensinada e praticada, como já demonstrado em um número substancial de evidências científicas, ainda é pouco difundido na maioria das instituições. Persistem muitos educadores e profissionais de saúde que ainda alimentam o equívoco de que a aquisição de habilidades de comunicação é um processo inato, uma espécie de dote ou arte trazida do berço por alguns ${ }^{1}$.

Dessa forma, a adequada formação de vínculo entre o profissional de saúde e o usuário, durante o aconselhamento, é necessária e favorece a troca de informações sobre o teste e a doença, identificando as preocupações e necessidades do indivíduo ${ }^{8}$.

Os profissionais envolvidos nesse processo precisam também ser sensíveis a reconhecer os estigmas relacionados ao HIV. A discriminação contra as pessoas que vivem com HIV é considerada uma das mais potentes barreiras à prevenção e ao tratamento e existe em diversos cenários, inclusive nos que envolvem cuidados em saúde ${ }^{21}$.

Sabe-se que um indicador do prognóstico ao nascimento é o acesso à assistência pré-natal e que os cuidados assistenciais no primeiro trimestre se refletem numa maior qualidade dos cuidados maternos. Nesse contexto, o diagnóstico da infecção pelo HIV dado no início da gestação possibilita melhor controle da infecção materna e melhores resultados de profilaxia da transmissão vertical do vírus. Por esse motivo, o teste anti-HIV oferecido à gestante ao iniciar o pré-natal é uma das estratégias usadas pelo Programa Nacional de IST/Aids para qualificar e ampliar o acesso ao diagnóstico do HIV ${ }^{9}$.

O momento do aconselhamento pré-teste é entendido como uma prática preventiva ampla que transcende o âmbito da testagem e contribui para a qualidade das ações educativas em saúde. Pressupõe-se que essa ferramenta de prevenção possa ser utilizada em vários momentos e serviços de saúde, não devendo restringir-se à oferta do exame anti-HIV. Dessa forma, deve ser um processo contínuo, inserido nas atividades das unidades básicas de saúde e articulado com os demais programas assistenciais existentes e com o cotidiano das práticas desenvolvidas pelos profissionais que atuam nos serviços de atenção primária à saúde ${ }^{22}$.

O serviço no qual foi feito o presente estudo realiza pré-natal de alto risco, ou seja, as gestantes diagnosticadas com alguma comorbidade são encaminhadas da atenção básica para esse ambulatório. Nele, é rotina realizar o aconselhamento e o teste anti-HIV na primeira consulta de prénatal, que geralmente é no primeiro trimestre, caso não tenham sido feitos na atenção básica, e repeti-los no terceiro trimestre da gestação. $\mathrm{O}$ aluno participa desse momento integrado ao seu estágio curricular.

No entanto, o que mais se constata na prática médica cotidiana, principalmente nas maternidades, é o mau uso do teste anti-HIV. Geralmente, ele é feito após o consentimento verbal da paciente, no momento em que se reconhece a necessidade de interná-la, sem proceder ao aconselhamento pré-teste, sem maiores explicações. O resultado é dado no ambiente habitual de urgência, sem privacidade, e, no máximo, o serviço de psicologia (se existir) é acionado quando o resultado é positivo. Talvez a frequente superlotação desses locais e o clima de urgência e estresse ali presentes dificultem que os profissionais proporcionem adequadamente essa assistência. Quando nesse ambiente estão inseridos indivíduos em formação, é provável que eles incorporem essa prática não conseguindo reconhecer a importância da realização do aconselhamento pré-teste HIV.

A possibilidade de replicar a consulta simulada aliada a um feedback formativo como estratégia na educação médica mostra-se com grande potencial de contribuir para promover no aluno a capacidade de autoavaliação e habilidade reflexiva. A experiência vivenciada com a fase II, permitindo que o discente "se veja fazendo" e compartilhando com ele a responsabilidade da avaliação, tem grande potencial para ser multiplicada em outras etapas da formação médica. Implicar o estudante no processo da avaliação formativa e estimular a identificação das próprias necessidades de mudança assume grande importância andragógica, tanto para o treinamento de habilidades práticas quanto para a promoção de transformações atitudinais.

\section{CONSIDERAÇÕES FINAIS}

Formar profissionais da saúde hábeis para a comunicação efetiva pressupõe a valorização e o desenvolvimento de estratégias de ensino e aprendizagem especificamente planejadas para oportunizar aos estudantes vivências relacionadas às habilidades de comunicação inseridas em seus currículos de graduação. Torna-se importante a utilização de estratégias na formação acadêmica que despertem a habilidades reflexiva sobre a necessidade de incrementar a habilidade de comunicação para aprimorar e qualificar o cuidado em saúde.

Conhecimentos e habilidades para a comunicação efetiva devem estar integrados na dinâmica da formação médica para que promovam atitudes positivas e facilitadoras da efetividade do cuidado com a saúde das pessoas.

Constata-se isso quando se observa que a estratégia empregada no estudo revelou que os alunos precisam de conhecimentos específicos e úteis para a qualidade do aconselhamento pré-teste do HIV e para a comunicação efetiva, a fim de que possam exercitar a habilidade de adequar tais informações à linguagem acessível para a grande maioria dos usuários dos sistemas de saúde.

Desse modo, é relevante que o preceptor desperte para a necessidade de incluir o aconselhamento na rotina do aprendiz e que a gestão do curso de Medicina dê suporte para a academia promover discussões, a fim de incrementar o currículo no quesito habilidade de comunicação. É importante que os componentes teóricos do curso sejam capazes de dar ênfase a esses aspectos, transversalmente, ao longo de todo o curso médico e de ressaltar sua aplicabilidade prática para a efetividade da comunicação médico-paciente.

\section{AGRADECIMENTOS}

A autora Larissa Cynthia Cesar Rodrigues agradece ao Instituto Santos Dumont o apoio institucional nas diversas fases de realização do estudo.

\section{REFERÊNCIAS}

1. Leite AJM, Caprara A, Coelho Filho JMC. Habilidades de comunicação com pacientes e famílias. São Paulo: Sarvie; 2007.

2. Medeiros FC, Pinheiro VGF, Leite AJM, Ribeiro MGF, Araújo MNT, Amaral AIA. Ensino de habilidades de comunicação a estudantes de Medicina: caminhos percorridos pela Faculdade de Medicina da Universidade Federal do Ceará, campus de Fortaleza. Cadernos ABEM, 2014;10:21-7.

\footnotetext{
REVISTA BRASILEIRA DE EDUCAÇÃo MÉDICA

8 (1) : e026; 2020
} 
3. Pagliosa FL, Da Ros MA. O relatório Flexner: para o bem e para o mal. Rev. bras. educ. méd 2008;32(4):492-9.

4. Laidlaw A, Hart J. Communication skills: an essential component of medical curricula. Part I: Assessment of clinical communication: AMEE Guide n 51. Med Teach 2011;33: 6-8.

5. ABIM Foundation, ACP-ASIM Foundation, European Federation of Internal Medicine. Medical professionalism in the new millennium: a physician charter. Ann Intern Med 2002;136(3):243-6.

6. Ministério da Educação. Conselho Nacional de Educação. Câmara de Educação Superior. Diretrizes Curriculares Nacionais do Curso de Graduação em Medicina. Brasília; 2014 [acesso em 22 set. 2018]. Disponível em: http://portal.mec.gov.br/index.php?option=com docman\&view=download \&alias=15874-rces003-14\&category_ slug=junho-2014-pdf\&Itemid=30192.

7. Cary J, Kurtz S. Integrating clinical communication with clinical reasoning and the broader medical curriculum. Patient Educ Coun 2013;92:361-5.

8. Brasil. Ministério da saúde. Diretrizes nacionais para o aconselhamento em DST/HIV/HV. Brasília; 2010 [acesso em 22 ago 2018]. Disponível em: http://www.aids.gov.br/sites/default/files/ anexos/page/2012/52294/teorizacao_o- ficina_acs.pdf.

9. Brasil. Ministério da Saúde. Secretaria de Vigilância em Saúde. Portaria $\mathrm{n}^{\circ} 34$, de 28 de julho de 2005. Regulamenta o uso de testes rápidos para diagnóstico de infecção pelo HIV em situações especiais. Diário Oficial da União 29 jul 2005.

10. Departamento de DST, Aids e Hepatites Virais. Portal sobre Aids, doenças sexualmente transmissíveis e hepatites virais [acesso em 20 maio 2019]. Disponível em: http://www.aids.gov.br.

11. Carneiro AJS. Aconselhamento na testagem anti-HIV no ciclo gravídico-puerperal: o olhar da integralidade. Ciênc. Saúde Colet. 2010;15(1):1216-26.

12. Fonseca PDL, Iriart, JAB. Aconselhamento em DST/Aids às gestantes que realizaram o teste anti-HIV na admissão para o parto: os sentidos de uma prática. Interface (Botucatu) 2012;16(41): 395-407.

13. Silva O, Tavares LHL, Paz LC. As atuações do enfermeiro relacionadas ao teste rápido anti-HIV diagnóstico: uma reflexão de interesse da enfermagem e da saúde pública. Enferm. em foco (Brasília) 2011;2(supl):58-62.

14. Brasil. Ministério da Saúde. Protocolo clínico e diretrizes terapêuticas para prevenção de transmissão vertical de HIV, sífilis e hepatite virais. Brasília; 2018 [acesso em 22 ago 2018]. Disponível em: http:// www.aids.gov.br/pt-br/pub/2015/protocolo-clinico-e-diretrizesterapeuticas-para-prevencao-da-transmissao-vertical-de-hiv.

15. Likert R. A technique for the measurement of attitudes. Archives of Psycology 1932;140:1-55.

16. Bardin L. Análise de conteúdo. Lisboa: Edições 70; 1977.
17. Farias JPQ, Franco A, Santos KP, Dourado I, Castro BG. Prevenção da transmissão vertical do HIV: atitude dos obstetras em Salvador, Brasil. Rev. Bras. Ginecol. Obstet. 2008;30(3):135-41.

18. Freitas Júnior RAO, Santos CAD, Lisboa LL, Freitas AKMS, Garcia VL, Azevedo GD. Incorporando a competência cultural para atenção à saúde materna em população quilombola na educação das profissões da saúde. Rev. bras. educ. méd. 2018;42(2):100-9 [acesso em 21 set 2018]. Disponível em: http://www.scielo.br/scielo.php?script=sci arttext\&pid=S010055022018000200100\&lng $=$ pt\&nrm $=$ iso.

19. Mangione S, Chakraborti C, Staltari G, Harrison R, Tunkel AR, Liou KT et al. Medical students' exposure to the humanities correlates with positive personal qualities and reduced burnout: a multi-institutional U.S. survey. JGIM 2018;33 (5):628-34.

20. Baile WK, Buckman R, Lenzi R, Glober G, Beale EA, Kudelka AP SPIKES - a six-step protocol for delivering bad news: application to the patient with cancer. Oncologist 2000;5(4):302-11.

21. Davtyan M, Olshansky EF, Lakon C. Adressing HIV stigma in health care: Strategies to address this potent barrier to treatment and prevention. Am J Nurs 2018;118(3):11.

22. Souza MCMR, Freitas MIF. Aconselhamento em HIV/Aids: representações dos profissionais que atuam na atenção primária à saúde. REME rev. min. enferm. 2012;16(1):18-24.

\section{CONTRIBUICCÃO DOS AUTORES}

Larissa Cynthia Cesar Rodrigues: idealizadora do estudo, participou da coleta, análise dos dados, redação e revisão final do texto.

Reginaldo Antônio de Oliveira Freitas Júnior: participou da coleta, análise e interpretação dos dados, bem como da redação e revisão final do texto.

Carolina Teixeira de Resende Barreto: participou da coleta, análise dos dados e revisão final do texto.

Alexandra do Nascimento Cassiano: contribuiu com o desenho do estudo e participou da coleta, análise e interpretação dos dados e da revisão final do texto.

Alexandra Silva de Lima: participou da coleta e análise dos dados.

Carla Glenda Souza da Silva: participou da coleta e análise dos dados.

\section{CONFLITO DE INTERESSES}

Os autores declaram não haver conflito de interesses neste estudo.

\section{ENDERECO PARA CORRESPONDÊNCIA}

Larissa Cynthia Cesar Rodrigues. Avenida Lima e Silva, 1536, Lagoa Nova, Natal, RN, Brasil, CEP: 59075-710.

E-mail: larissacrodrigues@yahoo.com.br 